\title{
Polarization asymmetry in CSS sources: Evidence of AGN fuel?
}

\author{
D. J. Saikia and N. Gupta
}

National Centre for Radio Astrophysics, TIFR, Post Bag 3, Ganeshkhind, Pune 411 007, India

Received 3 January 2003 / Accepted 16 April 2003

\begin{abstract}
The compact steep spectrum and gigahertz peaked spectrum sources are widely believed to be young radio sources, with ages $\lesssim 10^{6} \mathrm{yr}$. If the activity in the nucleus is fuelled by the supply of gas, one might find evidence of this gas by studying the structural and polarization characteristics of CSS sources as these evolve through this gas. We present polarization observations of a sample of CSS sources, and combine our results with those available in the literature, to show that CSS sources are more asymmetric in the polarization of the outer lobes compared with the more extended ones. We suggest that this could be possibly due to interaction of the jets with infalling material, which fuels the radio source. We also investigate possible dependence of the polarization asymmetry of the lobes on redshift, since this might be affected by more interactions and mergers in the past. No such dependence is found for the CSS sources, suggesting that the environments on the CSS scales are similar at different redshifts. However, the polarization asymmetry of the oppositely-directed lobes is larger at higher redshifts for the more extended sources, possibly reflecting the higher incidence of interactions in the past.
\end{abstract}

Key words. galaxies: active - quasars: general - galaxies: nuclei - radio continuum: galaxies

\section{Introduction}

There is a consensus of opinion that the compact steepspectrum (CSS) and gigahertz peaked spectrum (GPS) sources, defined to be $\leq 20 \mathrm{kpc}$ in a Universe with $q_{0}=0$ and $H_{0}=$ $100 \mathrm{~km} \mathrm{~s}^{-1} \mathrm{Mpc}^{-1}$, are young objects seen at an early stage of their evolution. Recent measurements of component advance speeds for a few very compact sources yield ages of about $10^{3}$ yr (Taylor et al. 2000; Polatidis \& Conway 2003), while spectral studies of CSS sources suggest ages $\$ 10^{5} \mathrm{yr}$ (Murgia et al. 1999). It is believed that the smallest CSS and GPS objects, christened the compact symmetric objects, or CSOs, evolve to the medium symmetric objects (MSOs) as the jets traverse outwards, and later on to the standard FRII radio sources (e.g. Carvalho 1985; Fanti et al. 1995; Readhead et al. 1996a,b; De Young 1997; O'Dea 1998 and references therein; Snellen et al. 2000; Perucho \& Martí 2002).

An interesting related question is the fuelling of these young radio sources, possibly due to the infall of gas to the central regions due to interacting companions and mergers. Detailed calculations (e.g. Hernquist \& Mihos 1995) showed that following a merger the infall of interstellar matter into the central few hundred pcs takes place over a time scale of $\approx 10^{8} \mathrm{yr}$. In such a situation, it should be possible to find evidence of this infalling material by following the evolution of the radio components in these CSS sources as they advance outwards and interact with this material. Their sizes and structures often appear to be affected by the ambient gas in the

Send offprint requests to: D. J. Saikia,

e-mail:djs@ncra.tifr.res.in central regions of the host galaxies (e.g. Spencer et al. 1991; Schilizzi et al. 2000), suggesting strong dynamical interaction with the external medium. The CSSs tend to be more asymmetric in both the brightness and location of the outer radio components compared with the larger sources (cf. Saikia et al. 1995, 2001; Arshakian \& Longair 2000). In a recent study of a sample of CSSs from the 3CR, S4, B2 and B3 samples it has been shown that the CSSs exhibit large brightness asymmetries with the flux density ratio for the opposing lobes being $>5$ for $\sim 25$ per cent of the objects, compared with only $\sim 5$ per cent for the objects of larger size (Saikia et al. 2002). The authors estimate the sizes of the clouds responsible for these asymmetries to be $\sim 3$ to $7 \mathrm{kpc}$, similar to those of dwarf galaxies (Swaters 1999), and speculate that such clouds might be responsible for the infall of gas which fuels the radio source.

Evidence of this gas may also be probed via polarization measurements (e.g. Saikia et al. 1987; van Breugel et al. 1992; Mantovani et al. 1994, 2002; Akujor \& Garrington 1995; Lüdke et al. 1998). In a double-lobed radio source, the component interacting with the dense gas should normally exhibit a higher rotation measure (RM) as well as depolarize more rapidly than the component advancing through the more tenuous medium. Although there have been relatively few studies of RM determinations in CSS and GPS objects, partly due to their low levels of polarization, such studies have sometimes revealed striking differences in the RM on opposite sides of the nucleus or high RM in regions where the jets bend sharply. For example, polarization observations of 3C147 show huge differential RMs between the two oppositely directed lobes, suggesting their evolution in an asymmetric environment 

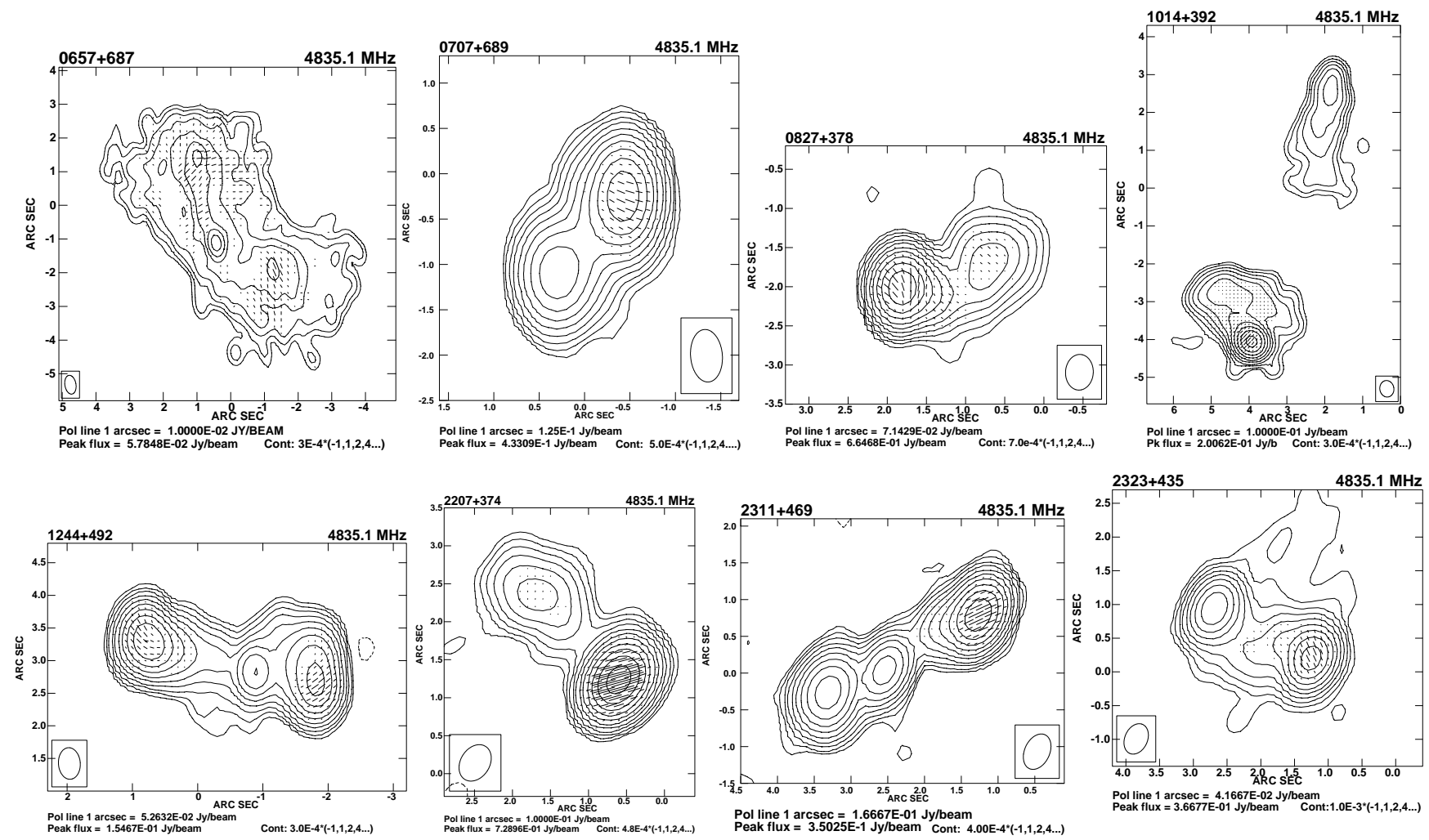

Fig. 1. Images of the CSS sources at $4835 \mathrm{MHz}$ with the polarization $\boldsymbol{E}$-vectors superimposed on the total-intensity contours. The peak brightness and contour levels in units of Jy/beam are given below each image.

(Junor et al. 1999). The quasar 3C216 has RMs in excess of $2000 \mathrm{rad} \mathrm{m}^{-2}$ in the arc of emission $\sim 140$ mas from the core where the jet bends sharply (Venturi \& Taylor 1999). The CSS quasar 3C43 has also been shown to have a high RM in the region where the jet bends sharply (Cotton et al. 2003).

In this paper, we use the polarization asymmetry, $r_{\mathrm{m}}$, defined to be the ratio of the degree of scalar polarization of the lobes on opposite sides of the nucleus at $\mathrm{cm}$ wavelengths $(\lambda 2$ to $6 \mathrm{~cm})$ to probe ambient gas asymmetries for both CSS and larger FRII radio sources. We present polarization observations of double-lobed CSS objects, and show that the CSS sources do tend to exhibit a higher polarization asymmetry than the larger FRII radio sources. We also show that while the polarization asymmetry for CSS objects does not depend on redshift, $r_{\mathrm{m}}$ increases with redshift for the more extended FRII sources. The implications of these results are discussed.

\section{The sample of sources}

The polarization of the lobes of the well-resolved, double-lobed CSS sources with a measured redshift and a size $\leq 20 \mathrm{kpc}$ from the S4 survey (Saikia et al. 2001) were determined from Very Large Array A-array observations at $4835 \mathrm{MHz}$. The data were calibrated and reduced using standard imaging and CLEANing procedures in the AIPS (Astronomical Image Processing System) package. All flux densities are on the Baars et al. (1977) scale. Excluding well-studied sources, such as 3C216 and 3C295, the images for the remaining sources are presented in Fig. 1, and some of their parameters are listed in
Table 1, which is arranged as follows. Column 1: source name in the IAU format using the B1950 coordinates; Cols. 2 to 4: the major and minor axes of the restoring beam in arcsec and its position angle (PA) in degrees; Col. 5: the rms noise in the images in units of $\mu \mathrm{Jy} /$ beam; Col. 6: component designation where $\mathrm{C}$ denotes the core or nuclear component; Col. 7: component flux densities, $S$, in units of mJy determined by integrating over a box around the component, except for the core components where the peak values are listed; Col. 8: the degree of polarization of the component, $m$. We estimate $\mathrm{m}$ following the procedure of Fanti et al. (2002), by blanking out points where the polarized intensity is $<3 \sigma$ in order to avoid spurious contributions of low signal-to-noise ratio polarization data. A fractional polarization image was then produced by excluding the totalintensity points which are also $<3 \sigma ; m$ represents the average value of fractional polarization while the upper limits represent about 3 times the error. The polarized intensity images were examined to ensure that a few unblanked pixels at the outer edges of a source do not lead to spurious values of $m$.

In addition to our observations, we have compiled from the literature the polarization information of the lobes of all CSSs which satisfy our selection criteria. Those sources with a high limit to the degree of polarization of one of the lobes, or with limits for both the lobes have not been included since it is difficult to get meaningful estimates of $r_{\mathrm{m}}$ for these objects. The final sample of 30 objects is listed in Table 2 which is arranged as follows. Column 1: source name in the IAU format using the B1950 co-ordinates; Col. 2: an alternative name; Col. 3: optical classification where $\mathrm{G}$ denotes a galaxy and $\mathrm{Q}$ a quasar; 
Table 1. The observational parameters and derived properties.

\begin{tabular}{|c|c|c|c|c|c|c|c|}
\hline \multirow[t]{2}{*}{ Source } & \multicolumn{3}{|c|}{ Beam size } & \multirow{2}{*}{$\begin{array}{c}\mathrm{rms} \\
\mu \mathrm{Jy} / \mathrm{b}\end{array}$} & \multirow[t]{2}{*}{$\mathrm{Cp}$} & \multirow{2}{*}{$\begin{array}{c}S \\
\mathrm{mJy}\end{array}$} & \multirow{2}{*}{$\begin{array}{l}m \\
\%\end{array}$} \\
\hline & " & " & $\circ$ & & & & \\
\hline \multirow[t]{3}{*}{$0657+68$} & 0.56 & 0.33 & 9 & 50 & S & 182 & 17.73 \\
\hline & & & & & $\mathrm{C}$ & 56 & $\lessgtr 0.50$ \\
\hline & & & & & $\mathrm{N}$ & 262 & 21.53 \\
\hline \multirow[t]{2}{*}{$0707+68$} & 0.58 & 0.35 & 5 & 165 & $\mathrm{~W}$ & 555 & 3.54 \\
\hline & & & & & $\mathrm{E}$ & 151 & $\lessgtr 0.55$ \\
\hline \multirow[t]{2}{*}{$0827+37$} & 0.46 & 0.35 & 177 & 110 & $\mathrm{~W}$ & 92 & 9.23 \\
\hline & & & & & $\mathrm{E}$ & 766 & 5.46 \\
\hline \multirow[t]{2}{*}{$1014+39$} & 0.44 & 0.37 & 10 & 75 & $\mathrm{~N}$ & 79 & $\lesssim 0.90$ \\
\hline & & & & & S & 421 & 11.71 \\
\hline \multirow[t]{3}{*}{$1244+49$} & 0.48 & 0.33 & 4 & 45 & $\mathrm{~W}$ & 229 & 5.98 \\
\hline & & & & & $\mathrm{C}$ & 40 & $\lesssim 0.50$ \\
\hline & & & & & $\mathrm{E}$ & 278 & 5.53 \\
\hline \multirow[t]{2}{*}{$2207+37$} & 0.51 & 0.38 & 148 & 110 & W & 779 & 8.41 \\
\hline & & & & & $\mathrm{E}$ & 53 & 5.82 \\
\hline \multirow[t]{3}{*}{$2311+46$} & 0.51 & 0.34 & 155 & 150 & W & 420 & 10.75 \\
\hline & & & & & $\mathrm{C}$ & 75 & $\lesssim 0.75$ \\
\hline & & & & & $\mathrm{E}$ & 229 & $\lessgtr 0.50$ \\
\hline \multirow[t]{2}{*}{$2323+43$} & 0.47 & 0.34 & 156 & 70 & $\mathrm{~W}$ & 544 & 1.94 \\
\hline & & & & & $\mathrm{E}$ & 360 & $\lessgtr 0.45$ \\
\hline
\end{tabular}

Col. 4: redshift; Col. 5: the projected linear size in kpc measured from the outermost peaks of radio emission; Col. 5: the wavelength of observation in $\mathrm{cm}$; Col. 6: the ratio of the degree of polarization of the oppositely-directed lobes, $r_{\mathrm{m}}$; Col. 7: references used for estimating these values.

For comparison with a sample of sources of larger linear sizes but observed at a similar wavelength, we have considered the $\lambda 6 \mathrm{~cm}$ observations of the sample of 47 sources by Garrington et al. (1991) to investigate the Laing-Garrington effect (Laing 1988; Garrington et al. 1988). This sample has a median linear size of $100 \mathrm{kpc}$ and a median redshift of 1 , with 40 of the sources being associated with quasars and 7 with radio galaxies. It is relevant to note that this sample was selected due to the presence of a radio jet and its degree of polarization asymmetry is likely to be higher than for a randomly selected sample, or one which has a larger proportion of galaxies (Holmes 1991). This selection effect will tend to diminish any possible difference in the degree of polarization asymmetry between the CSS and larger sources.

\section{Results and discussion}

The distribution of $r_{\mathrm{m}}$ for the entire sample of CSS objects (Fig. 2), shows that $\sim 50 \%$ of the sources appear to have $r_{\mathrm{m}}>5$, yielding a median value of $\sim 5$. There is a trend for the quasars to be more asymmetric than the galaxies which could be due to effects of orientation such as unresolved jets in some of the lobes. Also, there is a suggestion that the smallest CSS objects,
Table 2. The sample of CSS objects.

\begin{tabular}{|c|c|c|c|c|c|c|c|}
\hline Source & $\begin{array}{l}\text { Alt. } \\
\text { name }\end{array}$ & $\mathrm{O}$ & $z$ & $\begin{array}{c}l \\
\mathrm{kpc}\end{array}$ & $\begin{array}{c}\lambda \\
\mathrm{cm}\end{array}$ & $r_{\mathrm{m}}$ & Ref. \\
\hline $0127+233$ & $3 \mathrm{C} 43$ & $\mathrm{Q}$ & 1.459 & 16 & 3.6 & 1.8 & 1 \\
\hline $0221+276$ & $3 \mathrm{C} 67$ & $\mathrm{G}$ & 0.310 & 7.6 & 3.6 & $\gtrsim 27.8$ & 1 \\
\hline $0518+165$ & $3 \mathrm{C} 138$ & $\mathrm{Q}$ & 0.759 & 3.0 & 6.0 & $\gtrsim 40.0$ & 3 \\
\hline $0538+498$ & $3 \mathrm{C} 147$ & $\mathrm{Q}$ & 0.545 & 2.5 & 2.0 & 15.6 & 2 \\
\hline $0657+687$ & 4C68.07 & $\mathrm{G}$ & 0.110 & 5.6 & 6.0 & 1.2 & $\mathrm{P}$ \\
\hline $0707+689$ & 4C68.08 & $\mathrm{Q}$ & 1.141 & 6.3 & 6.0 & $\gtrsim 6.4$ & $\mathrm{P}$ \\
\hline $0827+378$ & $4 \mathrm{C} 37.24$ & $\mathrm{Q}$ & 0.914 & 5.9 & 6.0 & 1.7 & $\mathrm{P}$ \\
\hline $0858+292$ & $3 \mathrm{C} 213.1$ & $\mathrm{G}$ & 0.194 & 13 & 3.6 & 1.1 & 1 \\
\hline $0906+430$ & $3 \mathrm{C} 216$ & $\mathrm{Q}$ & 0.668 & 7.5 & 3.6 & 5.5 & 1 \\
\hline $1005+077$ & $3 \mathrm{C} 237$ & $\mathrm{G}$ & 0.877 & 6.9 & 3.6 & $\gtrsim 3.3$ & 1 \\
\hline $1014+392$ & $4 C 39.29$ & $\mathrm{G}$ & 0.206 & 16 & 6.0 & $\gtrsim 13.0$ & $\mathrm{P}$ \\
\hline $1153+317$ & 4C 31.38 & $\mathrm{Q}$ & 1.577 & 5.6 & 6.0 & 1.5 & 4 \\
\hline $1203+645$ & $3 \mathrm{C} 268.3$ & G & 0.371 & 4.6 & 3.6 & 21.4 & 1 \\
\hline $1244+492$ & $4 C 49.25$ & G & 0.206 & 6.0 & 6.0 & 1.1 & $\mathrm{P}$ \\
\hline $1250+568$ & $3 \mathrm{C} 277.1$ & $\mathrm{Q}$ & 0.321 & 5.2 & 3.6 & 11.5 & 1 \\
\hline $1350+316$ & $3 C 293$ & G & 0.045 & 2.2 & 3.6 & 25.6 & 1 \\
\hline $1409+524$ & $3 \mathrm{C} 295$ & G & 0.461 & 18 & 3.6 & 2.4 & 1 \\
\hline $1416+067$ & $3 C 298$ & $\mathrm{Q}$ & 1.439 & 9.0 & 3.6 & $\gtrsim 21.2$ & 1 \\
\hline $1443+773$ & 3C 303.1 & G & 0.267 & 4.7 & 6.0 & 3.0 & 3 \\
\hline $1447+771$ & 3C 305.1 & G & 1.132 & 13 & 3.6 & 1.9 & 1 \\
\hline $1458+718$ & 3C309.1 & $\mathrm{Q}$ & 0.904 & 11 & 3.6 & 2.1 & 1 \\
\hline $1517+204$ & $3 \mathrm{C} 318$ & G & 0.752 & 4.9 & 3.6 & $\gtrsim 7.7$ & 1 \\
\hline $2207+374$ & $4 \mathrm{C} 37.65$ & $\mathrm{Q}$ & 1.493 & 9.6 & 6.0 & 1.4 & $\mathrm{P}$ \\
\hline $2247+140$ & 4C14.82 & Q & 0.237 & 0.5 & 2.0 & 10.8 & 4 \\
\hline $2248+712$ & $3 \mathrm{C} 454.1$ & $\mathrm{G}$ & 1.841 & 10 & 3.6 & 1.2 & 1 \\
\hline $2249+185$ & $3 \mathrm{C} 454$ & $\mathrm{Q}$ & 1.757 & 4.2 & 6.0 & 23.1 & 3 \\
\hline $2252+129$ & $3 \mathrm{C} 455$ & G & 0.543 & 14 & 3.6 & 6.8 & 1 \\
\hline $2311+469$ & 4C46.47 & $\mathrm{Q}$ & 0.742 & 11 & 6.0 & $\gtrsim 21.5$ & $\mathrm{P}$ \\
\hline $2314+038$ & $3 \mathrm{C} 459$ & $\mathrm{G}$ & 0.220 & 19.5 & 6.0 & 19.8 & 5 \\
\hline $2323+435$ & $\mathrm{OZ}+438$ & $\mathrm{G}$ & 0.145 & 2.8 & 6.0 & $\gtrsim 4.3$ & $\mathrm{P}$ \\
\hline
\end{tabular}

References: 1: Akujor \& Garrington (1995), 2: Junor et al. (1999), 3: Lüdke et al. (1998), 4: van Breugel et al. (1984), 5: Thomasson et al. 2003, P: Present work.

with a linear size, say $<5 \mathrm{kpc}$, tend to exhibit a higher degree of polarization asymmetry, compared with the larger CSS objects.

The distribution of $r_{\mathrm{m}}$ for the control sample of larger sources is also shown in Fig. 2. The median value for the entire sample is $\sim 1.5$, with no significant difference between those with sizes either larger or smaller than the median value of $100 \mathrm{kpc}$. The median value of $r_{\mathrm{m}}$ for the 7 galaxies in the sample is also similar. Only 2 of the objects $(\sim 5 \%)$ exhibit a value of $r_{\mathrm{m}}>5$. These two, namely 0802+10 (3C191) and 1857+566, as well as $0017+15$ (3C9) with $r_{\mathrm{m}} \sim 4.7$ are dominated by prominent one-sided jets with a high degree of polarization (Lüdke et al. 1998; Saikia et al. 1983; 

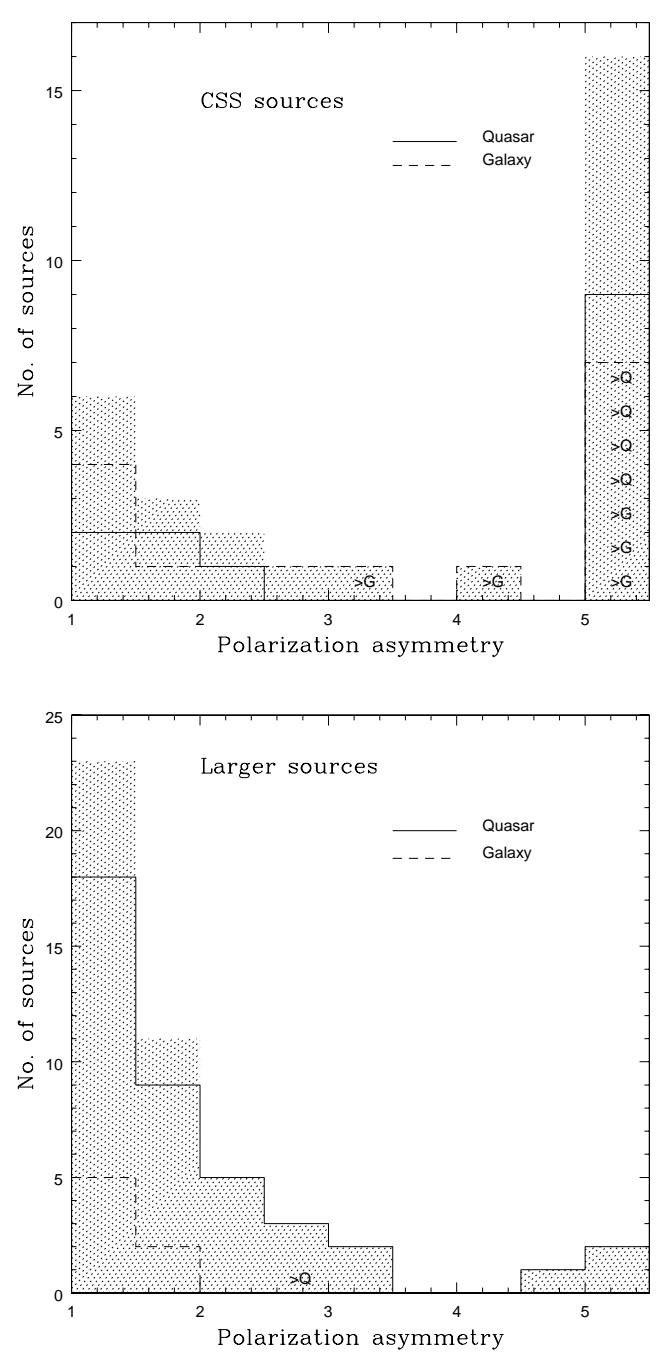

Fig. 2. The distributions of the polarization asymmetry parameter, $r_{\mathrm{m}}$ for the CSS and larger sources. All the sources with $r_{\mathrm{m}}>5$ have been placed in the last bin. The distributions for the entire sample are shown shaded.

Bridle et al. 1994). Such jets are highly dissipative with a more prominent hot-spot on the opposite side and are possibly intrinsically asymmetric in addition to effects of relativistic beaming (Saikia 1984).

The difference in the $r_{\mathrm{m}}$ distributions for the CSS and larger sources is very striking and highly significant. A Kolmogorov-Smirnov test shows the two populations to be different at a significance level of $>99.9 \%$. We have also confirmed this result by considering CSS and larger sources observed with a similar number of resolution elements along the source axes (median value $\sim 10$ ), by excluding 4 CSS and 5 larger objects which have been observed with less than 4 resolution elements. The tendency for CSS objects to have large values of $r_{\mathrm{m}}$ is also not due to one side of these objects being dominated by a prominent one-sided jet. Of the 16 sources with $r_{\mathrm{m}}>5$, approximately three-fourths of these have either no detected jets or only weak jets which do not contribute significantly to the polarized flux density of the lobes. Further, 7 of these 16 objects with $r_{\mathrm{m}}>5$ are associated with galaxies, which are expected to be at large angles to the line of sight and have at best weak radio cores and jets. This is consistent with the observations of these sources. It is also interesting to note that while some of the jets in CSS objects are strongly polarised at $\mathrm{cm}$ wavelengths such as in $3 \mathrm{C} 138$, there are also cases where the jets exhibit no significant polarised flux density as in 3C186, possibly due to Faraday depolarisation effects (Lüdke et al. 1998). A large sample of CSS objects which has been observed in both total intensity and linear polarization is the B3-VLA sample with $S(408) \geq 0.8 \mathrm{Jy}$ (Fanti et al. 2001). We have done a similar analysis for their sample using their listed average values of fractional polarization for the strongest components on opposite sides for the double-lobed sources. We again find a similar trend; the median value of the fractional polarization ratio is $\sim 3$ with approximately a third of the objects having a value $>5$.

This higher degree of polarization asymmetry in CSS objects could be due to interactions with dense clouds of gas which possibly fuel the radio source. The interactions of the jets with such clouds could either compress and shear magnetic fields, increasing the degree of polarization, or depolarise the emission due to Faraday effects. Both effects could contribute towards increasing the degree of polarization asymmetry of the oppositely-directed lobes. One would therefore not expect a significant correlation between the polarization and flux density asymmetries of the oppositely-directed lobes. The data also does not show a significant relation between these two parameters.

These dense clouds with sizes possibly similar to those of dwarf galaxies, are embedded in the large-scale halos with typical core radii of $\sim 100 \mathrm{kpc}$ which cause the Laing-Garrington effect. It is worth noting that these halos will cause only marginal polarization asymmetry on the scale of the CSS objects (Garrington \& Conway 1991). Also, sometimes it is the jet side which is more strongly depolarized and exhibit low levels of polarization as seen, for example, in 3C459 (Thomasson et al. 2003).

It is also of interest to enquire whether the polarization asymmetries depend on cosmic epoch because of the larger incidence of interactions and mergers in the past, as seen in Hubble Space Telescope studies of distant galaxies (cf. Abraham et al. 1996; Brinchmann et al. 1998; Ellis et al. 2000). The $r_{\mathrm{m}}$-redshift diagram for the CSS objects (Fig. 3) shows no significant dependence of $r_{\mathrm{m}}$ on redshift, although polarization observations of a larger number of sources at higher redshifts would be useful to confirm that this is indeed the case. The absence of a significant relationship suggests that although interactions and mergers may increase with redshift globally, the density asymmetries in the central regions of these active galaxies on the scale of the CSS objects, which are young and possibly still being fuelled by the infall of gas, are similar at different redshifts.

The $r_{\mathrm{m}}$-redshift diagram for the larger sources (Fig. 3) shows a clear trend for the polarization asymmetry to be higher at larger redshifts. Dividing the entire sample into the lowand the high-redshift ones at the median redshift of 1 , the median value of $r_{\mathrm{m}}$ is $\sim 1.4$ for the low-redshift sources and $\sim 2$ for the high-redshift objects. Leaving out the three objects 

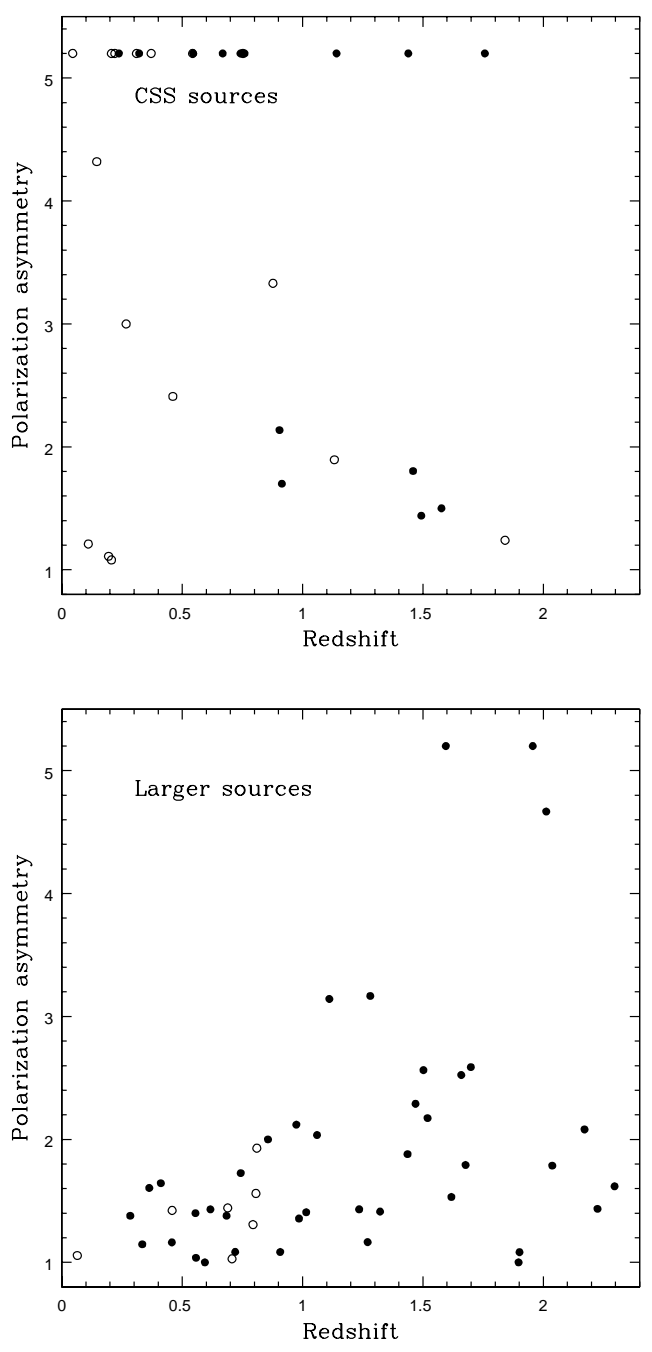

Fig. 3. The $r_{\mathrm{m}}$-redshift diagram for the CSS and larger sources. Those with $r_{\mathrm{m}}>5$ are shown at $r_{\mathrm{m}}=5.2$. The open and filled circles represent galaxies and quasars respectively.

with $r_{\mathrm{m}}>4.5$, reduces the median value for the high-redshift objects to $\sim 1.8$, which is still significantly higher than for the low-redshift sample. A Kolmogorov-Smirnov test shows the low- and high-redshift distributions to be different at a significance level of $>99$ percent. Excluding the three outlying jetdominated sources with $r_{\mathrm{m}}>4.5$ reduces the significance level to $\sim 98$ per cent. The number of resolution elements along the source axes for these sources are similar at different redshifts. The tendency for the high-redshift objects to exhibit a higher degree of polarization asymmetry could be due to interactions with companions on these scales. This would be consistent with the HST results mentioned earlier, and is also reminiscent of higher redshift radio sources appearing more distorted possibly due to interactions with companions (Barthel \& Miley 1988).

\section{Conclusions}

We present linear polarization observations of a sample of CSS sources, and show that the CSS sources are more asymmetric in the polarization of the outer lobes compared with those of the more extended ones. This could be possibly due to interaction of the jets with infalling material, which fuels the radio source. The polarization asymmetry of the lobes of CSS sources show no significant dependence on redshift, suggesting that the environments on the CSS scales are similar at different redshifts. However, the polarization asymmetry of the oppositely-directed lobes increases with redshift for the more extended sources, possibly reflecting the higher incidence of mergers and interactions in the past.

Acknowledgements. We thank Carla Fanti, the referee for several valuable comments and suggestions, and also for her very prompt refereeing of the paper. We also thank our colleagues and collaborators for their comments. The National Radio Astronomy Observatory is a facility of the National Science Foundation operated under cooperative agreement by Associated Universities, Inc. We have made use of the NASA/IPAC Extragalactic Database (NED), which is operated by the Jet Propulsion Laboratory, California Institute of Technology under contract with the National Aeronautics and Space Administration.

\section{References}

Abraham, R. G., Tanvir, N. R., Santiago, B. X., et al. 1996, MNRAS, 279, L47

Akujor, C. E., \& Garrington, S. T. 1995, A\&AS, 112, 235

Arshakian, T. G., \& Longair, M. S. 2000, MNRAS, 311, 846

Baars, J. W. M., Genzel, R., Pauliny-Toth, I. I. K., \& Witzel, A. 1977, A\&A, 61, 99

Barthel, P. D., \& Miley, G. K. 1988, Nature, 333, 319

Bridle, A. H., Hough, D. H., Lonsdale, C. J., Burns, J. O., \& Laing, R. A. 1994, AJ, 108, 766

Brinchmann, J., Abraham, R., Schade, D., et al. 1998, ApJ, 499, 112

Carvalho, J. C. 1985, MNRAS, 215, 463

Cotton, W. D., Spencer, R. E., Saikia, D. J., \& Garrington, S. T. 2003, A\&A, 403, 537

De Young, D. S. 1997, ApJ, 490, L55

Ellis, R. S., Abraham, R. G., Brinchmann, J., \& Menanteau, F. 2000, A\&G, 2, 10

Fanti, C., Fanti, R., Dallacasa, D., et al. 1995, A\&A, 302, 317

Fanti, C., Pozzi, F., Dallacasa, D., et al. 2001, A\&A, 369, 380

Garrington, S. T., Leahy, J. P., Conway, R. G., \& Laing, R. A. 1988, Nature, 331, 147

Garrington, S. T., Conway, R. G., \& Leahy, J. P. 1991, MNRAS, 250, 171

Garrington, S. T., \& Conway, R. G. 1991, MNRAS, 250, 198

Hernquist, L., \& Mihos, J. C. 1995, ApJ, 448, 41

Holmes, G. F. 1991, Ph.D. Thesis, University of Manchester

Junor, W., Salter, C. J., Saikia, D. J., Mantovani, F. M., \& Peck, A. B. 1999, MNRAS, 308, 955

Laing, R. A. 1988, Nature, 331, 149

Lüdke, E., Garrington, S. T., Spencer, R. E., et al. 1998, MNRAS, 299, 467

Mantovani, F., Junor, W., Fanti, R., Padrielli, L., \& Saikia, D. J. 1994, A\&A, 292, 59

Mantovani, F., Junor, W., Ricci, R., et al. 2002, A\&A, 389, 58

Murgia, M., Fanti, C., Fanti, R., et al. 1999, A\&A, 345, 769

O'Dea, C. P. 1998, PASP, 110, 493

Perucho, M., \& Martí, J. M. 2002, ApJ, 568, 639 
Polatidis, A. G., \& Conway, J. E. 2003, PASA, 20, 69

Readhead, A. C. S., Taylor, G. B., Xu, W., et al. 1996a, ApJ, 460, 612

Readhead, A. C. S., Taylor, G. B., Pearson, T. J., \& Wilkinson, P. N. 1996b, ApJ, 460, 634

Saikia, D. J. 1984, MNRAS, 208, 231

Saikia, D. J., Shastri, P., Cornwell, T. J., \& Banhatti, D. G. 1983, MNRAS, 203, 53P

Saikia, D. J., Singal, A. K., \& Cornwell, T. J. 1987, MNRAS, 224, 379

Saikia, D. J., Jeyakumar, S., Wiita, P. J., Sanghera, H. S., \& Spencer, R. E. 1995, MNRAS, 276, 1215

Saikia, D. J., Jeyakumar, S., Salter, C. J., et al. 2001, MNRAS, 321, 37

Saikia, D. J., Thomasson, P., Spencer, R. E., et al. 2002, A\&A, 391, 149
Schilizzi, R. T., Tschager, W., Snellen, I. A. G., et al. 2000, AdSpR, 26, 709

Snellen, I. A. G., Schilizzi, R. T., Miley, G. K., et al. 2000, MNRAS, 319, 445

Spencer, R. E., Schilizzi, R. T., Fanti, C., et al. 1991, MNRAS, 250, 225

Swaters, R. A. 1999, Ph.D. Thesis, Rijksuniversiteit Groningen

Taylor, G. B., Marr, J. M., Pearson, T. J., \& Readhead, A. C. S. 2000, ApJ, 541, 112

Thomasson, P., Saikia, D. J., \& Muxlow, T. W. B. 2003, MNRAS, 341, 91

van Breugel, W. J. M., Miley, G., \& Heckman, T. 1984, AJ, 89, 5

van Breugel, W. J. M., Fanti, C., Fanti, R., et al. 1992, A\&A, 256, 56

Venturi, T., \& Taylor, G. B. 1999, AJ, 118, 1931 